\title{
Heart failure: distinguishing failing myocardium from volume overload
}

\author{
Ronit H Zadikany ${ }^{1}$, TingTing Hong ${ }^{1,2}$ \& Robin M Shaw ${ }^{*, 1,2}$ \\ ${ }^{1}$ Smidt Heart Institute, Cedars-Sinai Medical Center, Los Angeles, 90048 CA, USA \\ ${ }^{2}$ Department of Medicine, Cedars-Sinai Medical Center \& UCLA, Los Angeles, 90048 CA, USA \\ *Author for correspondence: Tel.: +1 310967 3842; Fax: +1 3109673 891; robin.shaw@cshs.org
}
“By using CS as an additional biomarker to identify patients at an increased risk of future heart hospitalizations, clinical care can be better focused toward more aggressive surveillance or escalation of medical and advanced therapies."

First draft submitted: 14 April 2019; Accepted for publication: 26 April 2019; Published online: 7 June 2019

Keywords: biomarker $\bullet$ CBIN1 score $\bullet$ chronic heart failure $\bullet$ CS $\bullet$ natriuretic peptides

Heart failure is presently a major cause of morbidity and mortality in the USA and worldwide, with an everincreasing prevalence as our population ages. Despite promising improvements in our therapeutic interventions, only $50 \%$ of all patients with heart failure survive up to 4 years [1]. Furthermore, heart failure related hospitalizations and readmissions exact an unacceptably excessive burden on our healthcare system [2]. There is an urgent medical and economic need to identify patients with failing hearts early in their disease process and to improve clinical management of heart failure patients. Given the cost associated with heart failure-related hospitalization for outpatients with known ambulatory heart failure, there is enormous financial incentive to predict and therefore act to limit future hospitalizations.

Heart failure is not a disease but a complex multiorgan syndrome that fundamentally stems from an initial insult to cardiac function and then impairment of individual myocyte physiology. The syndrome of heart failure is progressive, involving a dynamic interplay between neurohormonal, inflammatory and biochemical changes that culminate in worsening muscle dysfunction and impaired systolic and/or diastolic function [3]. Our tools of assessment - such as history, physical examination, echocardiography, exercise tests and cardiac output measurements - focus on gross cardiac structure and hemodynamics. Structural measurements by echocardiography are not prognostic [4]. Other clinical assessment tools do not directly assess muscle health and are highly dependent on intracardiac volume, producing results that can change acutely with treatment. Temporal variation in functional measurements limits long-term prognostication [5].

Blood available biochemical marker testing has proven to be a valuable ancillary tool to aid in heart failure diagnosis, monitoring and even risk stratification. The natriuretic peptides, BNP and its N-terminal co-metabolite NT-proBNP, are the most established biomarkers used in clinical practice [6]. Both BNP and NT-proBNP are expressed and secreted as a response to increased myocardial wall tension and stress in the atria and ventricles, reflecting elevated cardiac filling pressures. BNP and NT-proBNP function as counter-regulatory hormones to angiotensin II, norepinephrine and endothelin, resulting in vasodilatory and diuretic effects [7]. The increased plasma concentrations of these hormones during volume and pressure overload make the natriuretic peptides useful biomarkers in establishing or repudiating the presence of elevated preload (and thus diagnosis of acute heart failure) in patients presenting with dyspnea in the acute setting [8]. Despite the success of natriuretic peptides as acute diagnostics, our country's growing management dilemma is not with acutely ill heart failure patients, but rather with those with known chronic and stable ambulatory heart failure.

Results of studies investigating the use of the natriuretic peptides to diagnose, risk stratify and guide treatment of patients with chronic heart failure are numerous, yet are not promising. The 2007 consensus guidelines discouraged use of natriuretic peptides in the initial evaluation of patients with suspected heart failure or for guiding therapy for patients with established heart failure [9]. After additional subsequent studies failed to show clear and consistent 
evidence for improvement in cardiovascular outcomes and mortality with natriuretic peptide-guided therapy in chronic heart failure, the 2017 updated guidelines consequently reduced the indication for using biomarkers in patients with chronic heart failure to a Class IIB strength of recommendation [10]. The limitations in diagnostic and prognostic accuracy of the natriuretic peptides can be partially attributed to noncardiac factors that have been shown to confound the interpretation of these assays. From false-negative values in obese and elderly patients to false elevation in patients with pulmonary hypertension and renal failure, precise assessment of natriuretic peptide levels in heart failure patients with a wide variety of comorbid conditions remains challenging. Additionally and most importantly, natriuretic peptides detect preload but not muscle health, thus by definition will follow a patient's fluid status that is usually dynamic and often not well controlled in the outpatient setting. Thus, for patients with chronic and stable ambulatory heart failure, volume-sensing natriuretic peptides have limited prognostic ability and are of limited additive use in standard clinical management.

The need exists for a biomarker that is able to reflect the intrinsic health of cardiac muscle cells independent of volume status. The cBIN1 score (CS), a blood test derived from the plasma concentration of a cardiomyocyte origin protein $\mathrm{cBIN} 1$, is a recently identified biomarker that serves as a blood-available indicator of cardiomyocyte heath and remodeling. cBIN1 is a cardiac-specific transverse tubule membrane sculpting protein, functioning to organize microdomains responsible for calcium release and excitation-contraction coupling [11,12]. cBIN1 is released from cardiomyocytes into the circulation, and the resultant cBIN1 blood level correlates with its content in myocardium [13,14]. Previous studies have demonstrated that the level of cBIN1 is not only reduced in animal models of heart failure, but also decreased in human biopsy samples from patients with end-stage cardiomyopathy [15-17]. The decreased transcription of cBIN1 in failing human hearts impairs excitation-contraction coupling and leads to impaired myocardial function [18].

The use of the CS, a dimensionless index based on the natural log inverse of plasma cBIN1 level, has been recently shown to perform well as a biomarker with the ability to diagnose and prognosticate patients with heart failure with preserved ejection fraction (HFpEF) [19]. When studied in ambulatory patients with HFpEF, CS values were more than two standard deviations higher in stable ambulatory patients with HFpEF compared with ageand sex-matched healthy volunteers as well as non-heart failure patients with confounding co-morbidities [19]. CS values were also markedly increased in ambulatory patients with New York Heart Association Class I and II symptoms whose NT-proBNP values were within the age-appropriate normal ranges [19]. These results indicate that an elevated CS value can be used as a marker to reflect intrinsic health of cardiac muscles independent of volume overload or acute decompensation.

The diagnostic and prognostic value of CS is complementary to that of the natriuretic peptides. By utilizing BNP and NT-proBNP as markers for myocardial wall stress and volume overload states in conjunction with CS as an indicator for cardiac myocyte failure, these two biomarkers can provide useful additive information in the assessment of chronic heart failure patients. The aforementioned clinical variables that affect accurate interpretation of the natriuretic peptides in clinical practice, such as renal function and body mass index, highlight the need for an additional parameter to assess myocardial health in heart failure patients with these co-morbidities. By using CS as an additional biomarker to identify patients at an increased risk of future heart hospitalizations, clinical care can be better focused toward more aggressive surveillance or escalation of medical and advanced therapies.

The use of CS is currently being studied in ambulatory patients with heart failure with reduced ejection fraction in order to assess whether CS is able to perform similarly well at prognosticating this patient population. Future potential advantages of CS include early preclinical diagnosis in asymptomatic patients, differentiating cardiogenic volume overload from extracardiac etiologies, predicting outcomes in stable ambulatory patients and evaluating myocardial recovery once on a successful therapeutic regimen. We look forward to the study of CS in different ambulatory healthcare settings.

\section{Author contributions}

All three authors participated in the concepts, research and writing of this review.

\section{Financial \& competing interests disclosure}

This work was supported by NIH grants HL094414 and HL138577 (RM Shaw), and HL133286 (T Hong). The authors have no other relevant affiliations or financial involvement with any organization or entity with a financial interest in or financial conflict with the subject matter or materials discussed in the manuscript apart from those disclosed.

No writing assistance was utilized in the production of this manuscript. 


\section{Ethical conduct of research}

We have obtained appropriate Cedars-Sinai institutional review board approval for all human investigations. In addition, for investigations involving human subjects, informed consent has been obtained from the participants involved.

\section{References}

1. Roger VL, Weston SA, Redfield MM et al. Trends in heart failure incidence and survival in a community-based population. JAMA 292(3), 344-350 (2004).

2. Rosamond W, Flegal K, Friday G et al. Heart disease and stroke statistics - 2007 update: a report from the American Heart Association Statistics Committee and Stroke Statistics Subcommittee. Circulation 115(5), e69-e171 (2007).

3. Jackson G, Gibbs CR, Davies MK, Lip GY. ABC of heart failure: pathophysiology. BMI 320(7228), 167-170 (2000).

4. Gackowski A, Isnard R, Golmard JL et al. Comparison of echocardiography and plasma B-type natriuretic peptide for monitoring the response to treatment in acute heart failure. Eur. Heart J. 25(20), 1788-1796 (2004)

5. Yancy CW, Jessup M, Bozkurt B et al. 2013 ACCF/AHA guideline for the management of heart failure: a report of the American College of Cardiology Foundation/American Heart Association Task Force on Practice Guidelines. J. Am. Coll. Cardiol. 62(16), e147-e239 (2013).

6. Sun RR, Lu L, Liu M et al. Biomarkers and heart disease. European Rev. Med. Pharmacol. Sci. 18(19), 2927-2935 (2014).

7. Chen HH, Burnett JC. Natriuretic peptides in the pathophysiology of congestive heart failure. Curr. Cardiol. Reports 2(3), 198-205 (2000).

8. Richards AM. The natriuretic peptides in heart failure. Basic Res. Cardiol. 99(2), 94-100 (2004).

9. Tang WH, Francis GS, Morrow DA et al. National Academy of Clinical Biochemistry laboratory medicine practice guidelines: clinical utilization of cardiac biomarker testing in heart failure. Clin. Biochem. 41(4-5), 210-221 (2008).

10. Yancy CW, Jessup M, Bozkurt B et al. 2017 ACC/AHA/HFSA focused update of the 2013 ACCF/AHA guideline for the management of heart failure: a report of the American College of Cardiology/American Heart Association Task Force on clinical practice guidelines and the Heart Failure Society of America. J. Card. Fail. 23(8), 628-651 (2017).

11. Hong T, Yang H, Zhang SS et al. Cardiac BIN1 folds T-tubule membrane, controlling ion flux and limiting arrhythmia. Nat. Med. 20(6), 624-632 (2014).

12. Hong TT, Smyth JW, Gao D et al. BIN1 localizes the L-type calcium channel to cardiac T-tubules. PLoS Biol. 8(2), e1000312 (2010).

13. Xu B, Fu Y, Liu Y et al. The ESCRT-III pathway facilitates cardiomyocyte release of cBIN1-containing microparticles. PLoS Biol. 15(8), e2002354 (2017).

14. Hong T, Shaw RM. Cardiac T-tubule microanatomy and function. Physiol. Rev. 97(1), 227-252 (2017).

15. Seidel T, Navankasattusas S, Ahmad A et al. Sheet-like remodeling of the transverse tubular system in human heart failure impairs excitation-contraction coupling and functional recovery by mechanical unloading. Circulation 135(17), 1632-1645 (2017).

16. Frisk M, Ruud M, Espe EK et al. Elevated ventricular wall stress disrupts cardiomyocyte T-tubule structure and calcium homeostasis. Cardiovasc. Res. 112(1), 443-451 (2016).

17. Hong TT, Cogswell R, James CA et al. Plasma BIN1 correlates with heart failure and predicts arrhythmia in patients with arrhythmogenic right ventricular cardiomyopathy. Heart Rhyth. 9(6), 961-967 (2012).

18. Fu Y, Shaw SA, Naami R et al. Isoproterenol promotes rapid ryanodine receptor movement to bridging integrator 1 (BIN1)-organized dyads. Circulation 133(4), 388-397 (2016).

19. Nikolova AP, Hitzeman TC, Baum R et al. Association of a novel diagnostic biomarker, the plasma cardiac bridging integrator 1 score, with heart failure with preserved ejection fraction and cardiovascular hospitalization. JAMA Cardiol. 3(12), 1206-1210 (2018). 
